\title{
The Rediscovery of the Social Side of Medicine: Philosophy and Value of the International Classification of Functioning, Disability and Health (ICF)
}

\author{
Type of article: conference Abstract
}

\author{
Wolfgang Seger \\ University of Bremen, Germany \\ Wolfgang.Seger@mdkn.de
}

\begin{abstract}
Medicine is at a risk to slide into a sole repair service for the malfunction of organs. But the patients' hopes and confidence toward doctors practicing this repair work go far beyond that: after acute medical treatment, many patients suffer from chronic impairments due to the natural course of disease or as a result of medical interventions. Despite the resulting handicaps, patients aim toward participating in family and social life, retaining a workplace, and receiving support to remain a valued member of family and community. Doctors should therefore not only concentrate on the natural science and technological part of medicine but also consider the background of their patients and their involvement in life situations, including environmental and personal factors, as these may influence functioning and disability as facilitators or barriers. Health insurances must organize, finance, and control the achievements of the post-acute treatment process with the goal of participation. Public health must combine and assess individual views to prepare reasonable population-based social, economic, and political decisions. The philosophy and structure of the International Classification of Functioning, Disability and Health (ICF) is supporting this attitude of medicine to complement the International Classification of Diseases (ICD) as a basis for health reports.
\end{abstract}

Keywords: Social Medicine, Bio - Medical Disease Model, Participation, Bio-PsychoSocial Disease Model, ICF

\section{Declaration of conflicts}

This article was presented at ICHSMT'16 as a key notes.

\section{Authors' biography}

Professor Dr. Wolfgang Seger is Professor of Rehabilitation Medicine, chairman of the German Federal Medical Rehabilitation Advisory Board, head of Social Medicine of all Social Health and Long-Term-Care Insurances in Lower Saxony, Germany

\section{References}

No reference 